MATEC Web of Conferences 22,02022 (2015)

DOI: $10.1051 /$ matec conf/ 20152202022

(C) Owned by the authors, published by EDP Sciences, 2015

\title{
Design and Analysis of an all-fiber MZI Interleaver Based on Fiber Ring Resonator
}

\author{
Huilan $\mathrm{Pu}^{*}$ \\ School of Electronic and Information Engineering, Lanzhou Jiaotong University, Lanzhou, Gansu, China \\ Huaiwei Lu \\ School of Mathematics and Physics, Lanzhou Jiaotong University, Lanzhou, Gansu, China
}

\begin{abstract}
An all-fiber Mach-Zehnder interferometer (MZI) interleaver using one planar $3 \times 3$ fiber coupler, one $2 \times 2$ fiber coupler and one 8 -shaped fiber ring resonator is developed by the new configuration. Based on its structure, the output spectrum expression is established and described by using the principle of fiber transmission and the matrix transfer function. The results of numerical simulation indicate that when the length difference of interference arms and the coupling coefficients of the couplers are some certain values, it obtains a uniform flat-top passband and similar to rectangular output spectrum. Compared with the traditional MZI interleaver, the isolation in stopband and the rolloff in transition band are strengthen, the $25 \mathrm{~dB}$ stopband bandwidth and $0.5 \mathrm{~dB}$ passband bandwidth are simultaneously remarkably improved. Compared with the asymmetrical ring resonator MZI interleaver, the influence of transmission loss on extinction ratio can be effectively reduced. The device has a certain ability to resist the deviation, which reduces the difficulties in fabricating it. The experiment results agree with the theoretical analysis well. The interleaver designed by the proposed approach has favorable performance, which has the potential application value in optical fiber communication system.
\end{abstract}

Keywords: interleaver; fiber ring resonator; fiber coupler; Mach-Zehnder interferometer(MZI); equivalent bandwidth

\section{INTRODUCTION}

In optical fiber communication system, with the demand of communication capacity increasingly growing, using optical fiber bandwidth efficiently is becoming more and more important. As the key device of dense wavelength division multiplexing (DWDM) system multiplexer/de-multiplexer is not only faced with the improvement of technical difficulty, device cost also increases accordingly. The optical interleaver has been proven to be an effective way to increase the capacity by doubling the number of channels ${ }^{[1-2]}$. The interleaver can not only increase the DWDM system multiplexing channel number, but also has solved the problem of device manufacturing technology.

Much study has been done about equivalent bandwidth and different bandwidth of interleavers. The main types of interleavers include methods of all-fiber Mach-Zehnder interferometer(MZI) [3-6] Gires-Tournoise interferometer ${ }^{[7-8]}$, Fabry-Perot interference filter(F-P) ${ }^{[9]}$, photonic crystal ${ }^{[10]}$ and so on. Among the abovementioned interleavers, all-fiber MZI interleaver based on fused fiber interferometer exhibits special qualities with even channel, simple structure and low insertion loss.

However, the conventional single-stage MZI interleaver of output spectrum is almost cosine, and their passband width and peak cannot satisfy the actual needs, therefore, the interleaver designed by cascading many MZI stages ${ }^{[11-12]}$ and a ring resonator with MZI(RRMZI) ${ }^{[13-14]}$ are presented. Theoretical analysis shows that the two plans may improve the transmission performance of MZI interleavers. But the cascaded MZI transmission performance is still not ideal. The upper and the lower interference arms of RRMZI are asymmetrical because the ring resonator is coupled to one arm of an MZI, and according to the interference theory, compensating for the transmission loss is needed in practice ${ }^{[13]}$.

In this paper, a novel all-fiber MZI interleaver is designed with one planar $3 \times 3$ fiber coupler, one $2 \times 2$ fiber coupler and an 8-shaped fiber ring resonator. Theoretical analysis and experimental verification indicate that designing structure parameters of the device reasonably can realize equivalent bandwidth transmission. The device of output spectrum is almost rectangular, common-mode rejection being high, crosstalk being low, the $25 \mathrm{~dB}$ stopband and $0.5 \mathrm{~dB}$ passband are increased remarkably. In addition, the influence of transmission loss on extinction ratio can also be effectively reduced. The device has a certain ability to resist the deviation, which reduces the difficulties in fabricating it.

*Corresponding author: phoebe168@126.com

This is an Open Access article distributed under the terms of the Creative Commons Attribution License 4.0, which permits unrestricted use, distribution, and reproduction in any medium, provided the original work is properly cited. 


\section{MATEC Web of Conferences}

\section{DEVICE STRUCTURE AND THEORETICAL ANALYSIS}

\subsection{Structure of an 8-shaped fiber ring resonator}

The 8-shaped fiber ring resonator is shown in Figure 1, which is composed of two $2 \times 2$ fiber couplers DC1 and $\mathrm{DC} 2$, which are linked together by fiber arm $l_{1}$ and $l_{2}$. The upper-left input port of DC2 and the lower-right output port of DC1 are connected by $l_{1}$, and the upper-right output port of DC2 and the lower-left input port of $\mathrm{DC} 1$ are connected by $l_{2}$. There is no cross connection point between fiber $l_{1}$ and $l_{2}$, and the optical signals through $l_{1}$ and $l_{2}$ are transmitted independently of each other.

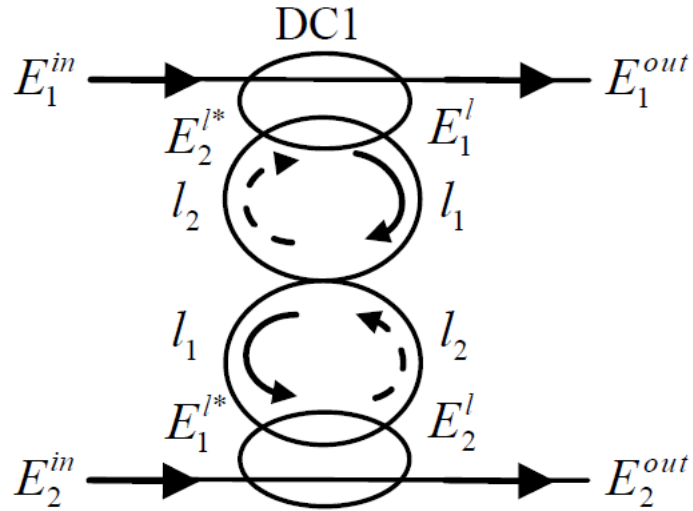

DC2

Figure 1. Structure of an 8-shaped fiber ring resonator

In the structure of 8-shaped fiber ring resonator, there are two input ports and two output ports, $k_{1}$ and $k_{2}$, representing the coupling coefficient of $\mathrm{DC} 1$ and $\mathrm{DC} 2 . \beta$ is the propagation constant in the fiber, and $\beta=2 \pi n_{\mathrm{eff}} / \lambda, n_{\mathrm{eff}}$ is the refractive index of the fiber, $\lambda$ is the wavelength. $\tau_{i}=\exp \left(-\alpha l_{i}\right) \quad(i=1,2) \quad(\alpha$ is the transmission loss coefficient) is the normalized loss of light signals through the transmission distance being $l_{1}$ and $l_{2}$. In Figure 1, [ $\left.E_{1}^{i n}, E_{2}^{i n}\right]$ are input light fields, and $\left[E_{1}^{\text {out }}, E_{2}^{\text {out }}\right]$ are output light fields, whereas the rest fields $E_{1}^{l}, E_{1}^{l^{*}}, E_{2}^{l}$ and $E_{2}^{l^{*}}$ are the circulated fields inside the fiber ring. By using the principle of fiber transmission and matrix transfer function $\mathrm{DC} 1, \mathrm{DC} 2$ and 8-shaped fiber ring resonator of output can be derived as follows in Eq.(1) Eq.(3):

$$
\begin{aligned}
& {\left[\begin{array}{l}
E_{1}^{\text {out }} \\
E_{1}^{l}
\end{array}\right]=\left[\begin{array}{cc}
\cos k_{1} & -\mathrm{j} \sin k_{1} \\
-\mathrm{j} \sin k_{1} & \cos k_{1}
\end{array}\right]\left[\begin{array}{l}
E_{1}^{\text {in }} \\
E_{2}^{l^{*}}
\end{array}\right]} \\
& {\left[\begin{array}{l}
E_{2}^{l} \\
E_{2}^{\text {out }}
\end{array}\right]=\left[\begin{array}{ll}
\cos k_{2} & -\mathrm{j} \sin k_{2} \\
-\mathrm{j} \sin k_{2} & \cos k_{2}
\end{array}\right]\left[\begin{array}{l}
E_{1}^{l^{*}} \\
E_{2}^{\text {in }}
\end{array}\right]}
\end{aligned}
$$

$$
\left[\begin{array}{l}
E_{1}^{\text {out }} \\
E_{2}^{\text {out }}
\end{array}\right]=\left[\begin{array}{ll}
C_{1} & C_{2} \\
C_{3} & C_{4}
\end{array}\right]\left[\begin{array}{l}
E_{1}^{\text {in }} \\
E_{2}^{\text {in }}
\end{array}\right]
$$

Where,

$E_{1}^{l^{*}}=\tau_{1} E_{1}^{l} \exp \left(-\mathrm{j} \beta l_{1}\right), E_{2}^{l^{*}}=\tau_{2} E_{2}^{l} \exp \left(-\mathrm{j} \beta l_{2}\right) ;$

$C_{1}=A^{-1}\left(\tau_{1} \tau_{2} \cos k_{2} \exp \left(-\mathrm{j} \beta\left(l_{1}+l_{2}\right)\right)-\cos k_{1}\right) ;$

$C_{2}=A^{-1} \tau_{2} \sin k_{1} \sin k_{2} \exp \left(-\mathrm{j} \beta l_{2}\right)$;

$C_{3}=A^{-1} \tau_{1} \sin k_{1} \sin k_{2} \exp \left(-\mathrm{j} \beta l_{1}\right)$;

$C_{4}=A^{-1}\left(\tau_{1} \tau_{2} \cos k_{1} \exp \left(-\mathrm{j} \beta\left(l_{1}+l_{2}\right)\right)-\cos k_{2}\right) ;$

$A=\tau_{1} \tau_{2} \cos k_{1} \cos k_{2} \exp \left(-\mathrm{j} \beta\left(l_{1}+l_{2}\right)\right)-1$.

\subsection{Structure of interleaver based on 8-shaped fiber ring resonator}

A novel all-fiber MZI interleaver based on 8-shaped fiber ring resonator is shown in Figure 2, which consists of one planar $3 \times 3$ fiber coupler DC0, one $2 \times 2$ fiber coupler DC3 and an 8-shaped fiber ring resonator, among which the DC0 is the fused planar $3 \times 3$ single mode fiber coupler in which three fibers in the same planar are weakly coupled, and it is used as input device where the function is distribution of the input light. When the coupling coefficient of DC0 is equal to $\pi / 2$ and the signal is input from the middle input port, the power coupling ratio is $0.5: 0.0: 0.5$ at the three output ports. Fiber couplers DC0 and DC3, DC3 and 8-shaped fiber ring resonator are connected in series by optical fiber $l_{3}$ and $l_{4}, l_{5}$ and $l_{6}$ respectively.

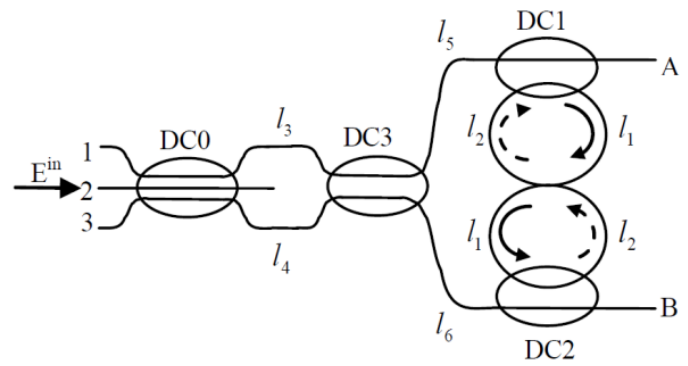

Figure 2. Structure of interleaver based on 8-shaped fiber ring resonator

Figure 2 shows the input light field $E^{\text {in }}$ through middle port of DC0 and output light fields from A and B ports of 8-shaped fiber ring resonator. Set $\mathbf{M}_{0}$ and $\mathbf{M}_{3}$ as the transmission matrix of fiber coupler DC0 and DC3 $\left(k_{0}\right.$ and $k_{3}$ represent their coupling coefficients), neglecting the transmission loss of fiber coupler, and we can obtain the output light fields $E_{A}$ and $E_{B}$ expressions for Eq.(5).

$\mathbf{M}=\mathbf{M}_{0} \cdot\left[\begin{array}{lll}0 & E^{\text {in }} & 0\end{array}\right]^{T}$ 


$$
\begin{aligned}
{\left[\begin{array}{c}
E_{A} \\
E_{B}
\end{array}\right]=} & {\left[\begin{array}{ll}
C_{1} & C_{2} \\
C_{3} & C_{4}
\end{array}\right]\left[\begin{array}{ll}
\exp \left(-\mathrm{j} \beta l_{5}\right) & 0 \\
0 & \exp \left(-\mathrm{j} \beta l_{6}\right)
\end{array}\right] \mathbf{M}_{3} } \\
& {\left[\begin{array}{ll}
\exp \left(-\mathrm{j} \beta l_{3}\right) & 0 \\
0 & \exp \left(-\mathrm{j} \beta l_{4}\right)
\end{array}\right]\left[\begin{array}{l}
\mathrm{M}(1) \\
\mathrm{M}(3)
\end{array}\right] }
\end{aligned}
$$

Where,

$M(1)$ and $M(3)$ represent the first line and the third line of matrix $\mathbf{M}$.

$\mathbf{M}_{0}=\left[\begin{array}{lll}\cos ^{2}\left(0.5 k_{0}\right) & \mathrm{j} 0.5 \sqrt{2} \sin k_{0} & -\sin ^{2}\left(0.5 k_{0}\right) \\ \mathrm{j} 0.5 \sqrt{2} \sin k_{0} & \cos k_{0} & \mathrm{j} 0.5 \sqrt{2} \sin k_{0} \\ -\sin ^{2}\left(0.5 k_{0}\right) & \mathrm{j} 0.5 \sqrt{2} \sin k_{0} & \cos ^{2}\left(0.5 k_{0}\right)\end{array}\right]$

$\mathbf{M}_{3}=\left[\begin{array}{cc}\cos k_{3} & -\mathrm{j} \sin \mathrm{k}_{3} \\ -\mathrm{jsink}_{3} & \cos k_{3}\end{array}\right]$

By the light intensity formula $P=E E^{*}, P_{A}$ and $P_{B}$ are normalized output intensity which are shown in Eq.(6).

$$
\left\{\begin{aligned}
P_{A}= & a_{0}+a_{1} \sin \beta\left(l_{3}-l_{4}\right)+a_{2} \cos \beta\left[\left(l_{3}-l_{4}\right)\right. \\
& \left.+\left(l_{5}-l_{6}\right)\right]+a_{3} \cos \beta\left[\left(l_{3}-l_{4}\right)-\left(l_{5}-l_{6}\right)\right] \\
P_{B}= & b_{0}+b_{1} \sin \beta\left(l_{3}-l_{4}\right)+b_{2} \cos \beta\left[\left(l_{3}-l_{4}\right)\right. \\
& \left.+\left(l_{5}-l_{6}\right)\right]+b_{3} \cos \beta\left[\left(l_{3}-l_{4}\right)-\left(l_{5}-l_{6}\right)\right]
\end{aligned}\right.
$$

Where,

$$
\begin{aligned}
& a_{0}=0.5 \sin ^{2} k_{0}\left(C_{1}^{2}+C_{2}^{2}\right) ; \\
& a_{1}=\left(C_{1}^{2}-C_{2}^{2}\right) \sin ^{2} k_{0} \sin k_{3} \cos k_{3} ; \\
& a_{2}=C_{1} C_{2} \sin ^{2} k_{0} \cos ^{2} k_{3} ; \\
& a_{3}=C_{1} C_{2} \sin ^{2} k_{0} \sin ^{2} k_{3} ; \\
& b_{0}=0.5 \sin ^{2} k_{0}\left(C_{3}^{2}+C_{4}^{2}\right) ; \\
& b_{1}=\left(C_{3}^{2}-C_{4}^{2}\right) \sin ^{2} k_{0} \sin k_{3} \cos k_{3} ; \\
& b_{2}=C_{3} C_{4} \sin ^{2} k_{0} \cos ^{2} k_{3} ; \\
& b_{3}=C_{3} C_{4} \sin ^{2} k_{0} \sin ^{2} k_{3} .
\end{aligned}
$$

In Figure 2, the interleaver based on 8-shaped fiber ring resonator is symmetry structure. Analysis of Eq.(6) shows that when the power coupling ratio of DC0 is $0.5: 0.0: 0.5$, and DC3 is $3 \mathrm{~dB}$ fiber coupler, DC1 and DC2 have the same coupling coefficient, which will obtain symmetric and complementary wavelength response at the both output ports. When $k_{1}=k_{2}=k, k_{0}=\pi / 2, k_{3}=\pi / 4$, through the $q_{3}=2 l_{4}, l_{5}=2 l_{4}, l_{6}=l_{4}, l_{3}, l_{4}^{2}=3 l_{4}$, $\theta=\beta \Delta l$ (transmission phase delay of interference arm), if the transmission loss of 8-shaped fiber ring resonator is ignored, Eq.(6) will be simplified to Eq.(7).

$\left\{\begin{array}{l}P_{A}=\frac{1}{2}-\frac{\left(\sin ^{4} k-2 \cos ^{2} k-2 \cos ^{2} k \sin 4 \theta\right) \sin \theta}{2\left(\sin ^{4} k+2 \cos ^{2} k-2 \cos ^{2} k \cos 4 \theta\right)} \\ P_{B}=\frac{1}{2}+\frac{\left(\sin ^{4} k-2 \cos ^{2} k-2 \cos ^{2} k \sin 4 \theta\right) \sin \theta}{2\left(\sin ^{4} k+2 \cos ^{2} k-2 \cos ^{2} k \cos 4 \theta\right)}\end{array}\right.$

It can be seen from Eq.(6) that $P_{A}$ and $P_{B}$ contain transmission phase delay of interference arm and coupling coefficients of fiber couplers. The former decides cycle of the output spectrum, and the later determines waveform of the output spectrum. When the interference arm length and the coupling coefficients select appropriate values, the device can realize comb and equivalent bandwidth of output spectrum. In addition, through numerical calculation Eq.(7), extreme value point and maximum channel segregation can determine $k=\pi / 2.25$.

\section{NUMERICAL SIMULATION AND DISCUS- SION}

\subsection{Numerical Simulation}

Application of optimization algorithm, without regard to transmission loss $(\tau=1)$, the simulation result is shown in Figure 3 (In the following figure, $P_{A}$ is the solid line, $P_{B}$ is dotted line.), which is calculated with the parameters $k_{0}=\pi / 2, k_{3}=\pi / 4$ $k_{1}=k_{2}=\pi / 2.25, \lambda_{0}=1550 \mathrm{~nm}, \Delta l=2 \mathrm{~mm}$ and $n_{\text {eff }}=1.457$. Figure 3 shows, interleaver output spectrum of the port A and B are the same two groups of bandwidth periodic spectral lines, and their frequency interval is $100 \mathrm{GHz}$. Flat-top can offset the negative influence of channel wavelength drift.

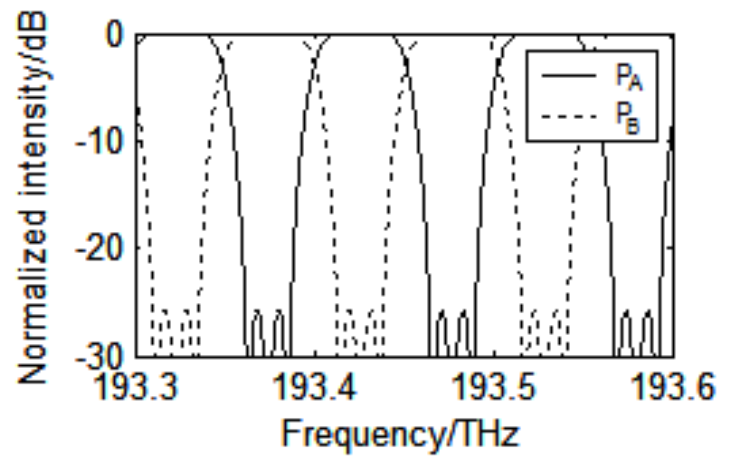

Figure 3. Normalized intensity of all-fiber MZI interleaver with $k_{0}=\pi / 2, k_{1}=k_{2}=\pi / 2.25, k_{3}=\pi / 4$

It is clear in Figure 3 that the proposed interleaver is attractive in flat stopband/passband and big isolation, and the $25 \mathrm{~dB}$ stopband and $0.5 \mathrm{~dB}$ passband are 26.2GHz and 38.4GHz. In Ref.[14], conventional MZI interleaver's output spectrum is similar to cosine wave, 


\section{MATEC Web of Conferences}

and the $25 \mathrm{~dB}$ stopband and $0.5 \mathrm{~dB}$ passband are $3.6 \mathrm{GHz}$ and $21.3 \mathrm{GHz}$. In Ref.[11], MZI interleaver is cascaded, and the $25 \mathrm{~dB}$ stopband and $0.5 \mathrm{~dB}$ passband are $15.8 \mathrm{GHz}$ and $30.2 \mathrm{GHz}$. By comparison, the novel interleaver through 8-shaped fiber ring resonator phase adjustment and the output spectrum produces curve steep edge and is more close to the rectangular wave, and the $25 \mathrm{~dB}$ stopband and $0.5 \mathrm{~dB}$ passband are wider than others.

3.2 Influence of Coupling Coefficient on the Interleaver Response
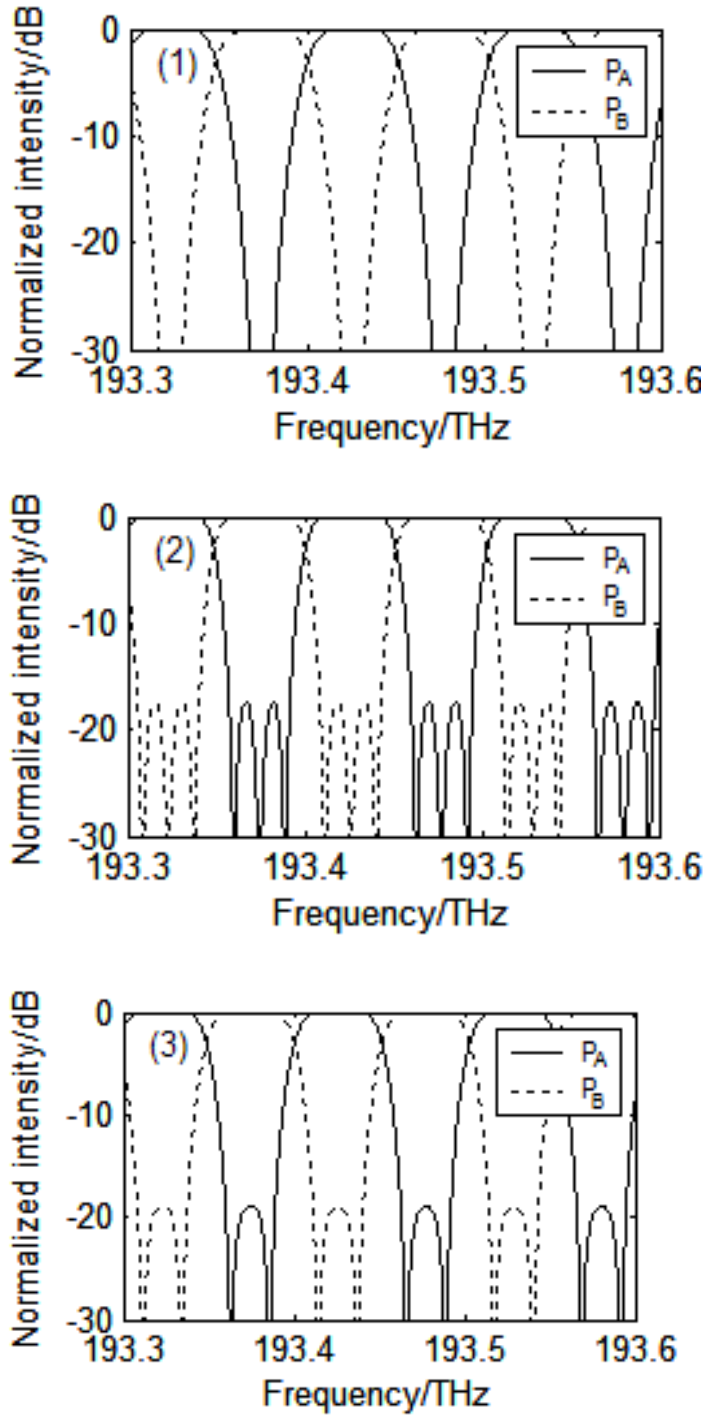

Figure 4. Normalized intensity of all-fiber MZI interleaver

$$
\begin{aligned}
& \text { with } k_{0}=\pi / 2, k_{3}=\pi / 4, \quad \text { (1) } k_{1}=k_{2}=k+\Delta k \\
& k_{1}=k_{2}=k-\Delta k, \text {,(3) } k_{1}=k-\Delta k, k_{2}=k+\Delta k
\end{aligned}
$$

Eq.(7) shows that when the coupling coefficients of DC0 and DC3 are constant, the length differences of interference arms are constant, and the output spectrum mainly depends on coupling coefficients of DC1 and DC2. They will determine the size of the channel segregation and shape of output spectrum. In the process of experiment, the experimental environment and production process will influence ideal value of coupling coefficient and there is a deviation. Set $\Delta k=k_{i} \times 5 \%(i=1,2)$ as the deviation of coupling coefficient, the numerical simulation results show the deviation in range. If the coupling coefficients of DC1 and DC2 increase simultaneously, the $25 \mathrm{~dB}$ stopband will change the range of $21.1 \sim 26.2 \mathrm{GHz}$, and the $0.5 \mathrm{~dB}$ passband will change the range of $35.5 \sim 38.4 \mathrm{GHz}$. Figure 4(1) is the output spectrum of interleaver with $k_{1}=k_{2}=k+\Delta k$, its stopband and passband compared with the best value decrease slightly, and its side-lobe level decreases. If the coupling coefficients of DC1 and DC2 reduce simultaneously, the $25 \mathrm{~dB}$ stopband will change the range of $26.2 \sim 29.4 \mathrm{GHz}$, and the $0.5 \mathrm{~dB}$ passband will change the range of $38.4 \sim 40.9 \mathrm{GHz}$. Figure 4(2) is output spectrum of interleaver with $k_{1}=k_{2}=k-\Delta k$, and its stopband and passband compare with the best value increase slightly, but the side-lobe level also increases. If the coupling coefficient of DC1 reduces and the coupling coefficient of DC2 increases, the output spectrum will basically remain unchanged, while side-lobe will change slightly. Figure 4(3) is output spectrum of interleaver with $k_{1}=k+\Delta k$ and $k_{2}=k-\Delta k$, the $25 \mathrm{~dB}$ stopband is $26.24 \mathrm{GHz}$, and the $0.5 \mathrm{~dB}$ passband is $38.27 \mathrm{GHz}$. Because of the structure symmetry, when the coupling coefficient of DC1 increases and the coupling coefficient of DC2 reduce, the simulated results are consistent with Figure 4(3).

By analyzing the interleaver output spectrum performance, it can be concluded that when slight deviation of the coupling coefficients of DC1 and DC2 exists, the $25 \mathrm{~dB}$ stopband and $0.5 \mathrm{~dB}$ passband will show deviation, but the change is not obvious, and the channel segregation can be above $30 \mathrm{~dB}$, which can satisfy the actual needs. It also indicates that the performance can be controlled in 5\% deviations during the fabrication, which reduces the difficulties in fabricating the all-fiber MZI interleaver.

\subsection{Influence of Trasmission Loss on the Interleaver Response}

In the discussion about transmission performance of interleaver, the transmission loss of optical signal in the 8-shaped fiber ring resonator is ignored. But the fiber must be curved by the fiber ring so that fiber transmission loss will certainly be introduced. In order to analyze influence of transmission loss on interleaver response, ${ }_{\tau}$ choose different normalized loss value $\tau_{1}=\tau_{2}=\sqrt{\tau}$ to simulate calculation. In Fig5.(1), the peak of output spectrum with $\tau=0.9$, is descended by about $0.35 \mathrm{~dB}$ and that with $\tau=0.8, k_{1}=k_{2}=k$ is descended by about $0.76 \mathrm{~dB}$ in Figure 5(2). Compared with asym- 
metric optical fiber auxiliary ring structure in Ref.[13], the interleaver based on 8-shaped fiber ring resonator is proposed in this paper, and there are no amplitude differences about the two interference beams, and the influence of transmission loss on extinction ratio can be effectively reduced.
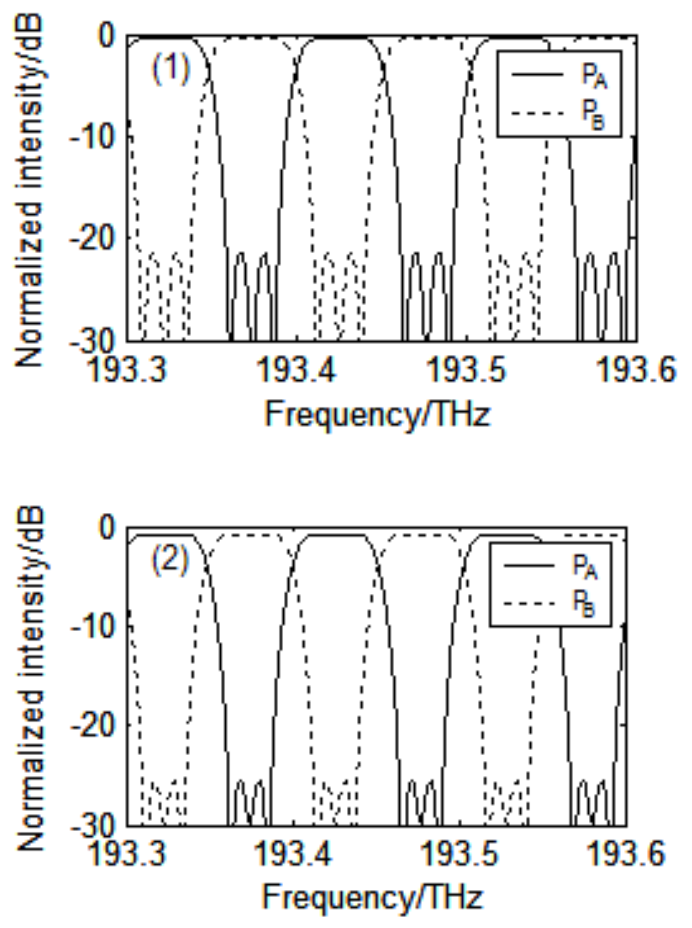

Figure 5. Normalized intensity of all-fiber MZI interleaver with (1) $\quad k_{1}=k_{2}=k-2 \% \times k, \tau=0.9$ $k_{1}=k_{2}=k, \tau=0.8$

\section{EXPERIMENTAL APPARATUS}

The basic principle of interleaver is the light interference. Because the optical fiber welding will introduce waveguide discontinuity, which will affect insertion loss and polarization of optical fiber, the continuous melting method should be used. In the process of melt control, the compute test system will monitor the change of couplers ratio and interference arm's length. First, according to the conventional method to melt $3 \mathrm{~dB}$ fiber coupler DC3, from one end of the DC3 insert another optical fiber continuous melting pull DC0, and then from the other end of the DC3 fusion DC1 of 8 -shaped fiber ring resonator, to the two synthetic fibers drawn from the DC3 insert another optical fiber. Inserted fiber and DC3 draw one fiber to fuse coupler DC1, and the output port of inserted fiber and DC3 draws another optical fiber to fuse coupler DC2. When couplers coupling ratio and interference arm's length meet the requirement, inserted fiber will weld in DC2 out end with DC1 insert end. The method of melting drawn by fiber interference arms and fiber couplers can refer to Ref. [15-16].

The output performance of the experimental interleaver is tested, light source is Santec company TSL2210 wavelength tunable laser, and wavelength range is $1520 \sim 1580 \mathrm{~nm}$. The linear polarized light is input through middle port of $\mathrm{DC} 0$, its power is $1 \mathrm{~mW}$, and port $\mathrm{A}$ and $\mathrm{B}$ of ring resonator measure the output power. Figure 6 is tested output of spectrum of the experimental sample. Compared with Figure 3, the influence of experimental environment makes micro variable exists in the experimental sample output spectrum, and the peak also declines slightly because of transmission loss. Overall experimental sample spectrum agrees with theoretical analysis well.

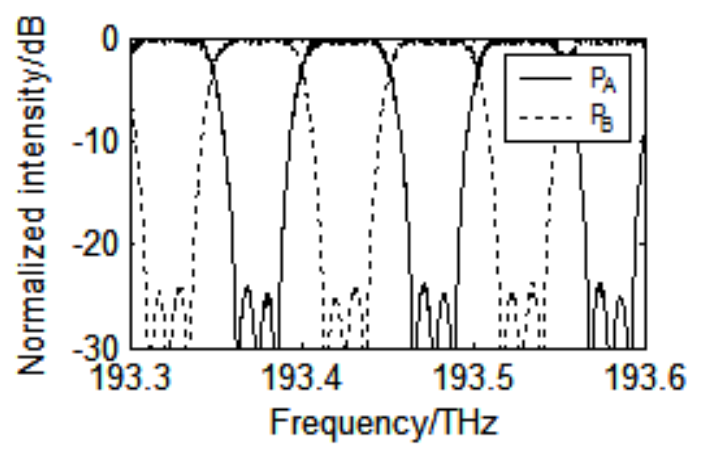

Figure 6. Output spectra of the interleaver from experiment.

\section{CONCLUSION}

The all-fiber MZI interleaver based on 8-shaped fiber ring resonator is proposed in this paper, and its structure parameters are obtained and described by theoretical analysis and numerical simulation, which indicates that when the length difference of interference arms and the coupling coefficient of the couplers are some certain values, the $25 \mathrm{~dB}$ stopband and 0.5 passband are improved remarkably, which can achieve almost rectangular spectrum response, and the influence of transmission loss on extinction ratio can reduce remarkably. The experimental results agree with the theoretical analysis. The interleaver designed by the proposed approach has favorable performance, which has the potential application value in optical fiber communication system. The study may provide reference for the facture of the apparatus later.

\section{ACKNOWLEDGEMENT}

This study is supported by the Natural Science Foundation of GanSu Province of China (No.1310RJZA075). 


\section{MATEC Web of Conferences}

\section{REFERENCES}

[1] X. H. Ye, M. Zhang \& P. D. Ye. 2006. Flat-top interleavers with chromatic dispersion compensator based on phase dispersive free space Mach-Zehnder interfereometer. Opt. Commun. 2: 255.

[2] Naveen Kumar, M. R. Shenoy \& B. P. Pal. 2008. Flattop all-fiber wavelength interleaver for DWDM transmission: design analysis, parameter optimization, fabrication and characterization recipe. Opt. Commun. 20: 5156.

[3] H. W. Lu, K. J. Wu, Y. Wei, B. G. Zhang \& G. W. Luo. 2012. Study of all- fiber asymmetric interleaver based on two stage cascaded Mach-Zehnder Interferometer. Opt. Commun. 6: 1118 .

[4] H. W. Lu. 2013. Study of a Novel All-fiber $3 \times 3$ Interleaver with a Dual-eight-shaped Ring Resonator. IEEE Photo. Tech. Lett. 9: 806.

[5] H. W. Lu, Y. Wei, K. J. Wu, B. G. Zhang \& G. W. Luo 2011.Design of all- fiber Asymmetric Interleaver with $2 \times 2$ and $3 \times 3$ Fiber Couplers. Acta Optica Sinica, $11,1106002-1$

[6] H. L. Pu \& H. W. Lu. 2014. Design of a New Type of All-fiber MZI Interleaver with Different Bandwidth. Journal of Lanzhou Jiaotong University, 3: 120

[7] X. L. Wang, W. C. Huang, Y. Zhang \& Z. P. Cai. 2009. Design and analysis on a Gires-Tournois resonator based interleaver. Opt. Lett. 1, 51.

[8] L. Wei \& J. W. Y. Lit. 2007. Design optimization of flattop interleaver and its dispersion. Opt. Express. 10 6439.

[9] Q. Ye, R. H. Qu \& Z. J. Fang. 2007. Generation of millimeter-wave sub-carrier optical pulse by using a Fabry-Perot interferometer. Chin. Opt. Lett., 1: 8.

[10]X. X. Pan, F. G. Luo \& L. Deng. 2011. Structure design of interleaver based on birefringent-crystals. Asia Commun. Photonics Conf. Exhib., ACP.

[11]S. W. Kok, Y. Zhang, C. Y. Wen \& Y. C. Soh. 2003. Design of all-fiber optical interleavers with a given specification on passband ripples. Opt. Commun. 226: 241

[12]Y. Yu, J. J. Dong, X. Li \& X. L. Zhang. 2011. Ultra-wideband generation based on cascaded mach-zehnder modulators. IEEE Photo. Tech. Lett. 23: 1754.

[13]X. W. Dong, L. Pei, O. Xu, S. H. Lu, S. C. Feng, R. F. Zhao \& Z. W. Tan. 2008. Study of Interleaver Based on Ring Resonator Assisted Mach-Zehnder Interferometer. Acta Optica Sinica, 4: 638.

[14]W. B. Li \& J. Q. Sun. 2008. Analysis of Characteristics of the Interleaver Based on a Double-Coupler Resonator. Chinese J. Laser, 8: 1191.

[15]W. M. Sun, S. Shi \& Q. Dai. 2009. Fabrication and measurement of tapered fibers. Journal of Optoelectro -nics Laser, 11: 1474.

[16] S. W. Harum, K. S. Lim, A. A. Jasim \& H. Ahmad, 2010. Fabrication of tapered fiber based ring resonator. Laser Phys. 7: 1629 